\title{
POLEMICAS
}




\section{ETNOGRAFIA Y POLITICA EDUCATIVA *}

Leonor Zubieta $V^{*}$

El objeto de esta intervención es el de tratar de dar respuesta, aunque de manera muy sintética, a la pregunta: ¿Qué puede aportar la investigación educativa orientada por una perspectiva etnográfica a los procesos de formulación e implementación de política educativa?

Con ese propósito, la exposición se desarrollará en el siguiente orden:

1. Se hará una rápida consideración de lo que se ha entendido y se entiende por investigación etnográfica en educación de sus antecedentes a nivel internacional y de las experiencias que al respecto se adelantan en el país.

2. En segundo lugar, se procurará establecer una comparación entre otras perspectivas investigativas muy generalizadas en nuestro medio y en el campo educativo, y la perspectiva etnográfica, en términos de las conceptualizaciones teóricas que manejan, los métodos y técnicas que emplean, la naturaleza y la utilidad de la información que proporcionan y el tipo de problemas que permiten plantear y resolver.

3. Se intentará, por último, precisar la participación que tiene o que puede llegar a tener la investigación etnográfica en los procesos de determinación de política y de toma de decisiones para el sector educativo colombiano.

\section{Etnografía y Educación}

En sus orígenes antropológicos, la Etnografía se interpretó, y aún se interpreta en algunos ámbitos específicos de esa disciplina, como el procedimiento de observación y de descripción intensiva de una tribu o etnia particular. Como propósito general, se le definió el de procurar la recopilación más completa y exacta posible de la información necesaria para reconstruir la cultura y conocer los fenómenos sociales propios de tales grupos sociales, considerados entonces como "pueblos primitivos"2.

Los desarrollos posteriores de la Antropología significaron cambios en la concepción tanto teórica como metodológica del trabajo etnográfico. La incidencia del funcionalismo condujo a la superación de su formulación inicial: De la "mera descripción" de una serie de instituciones sociales que, se suponía, conformaban de manera independiente y aislada la realidad cultural y social del pueblo estudiado, se llegó a la definición del estudio "integral" de la sociedad como objeto propio de la Etnografía, entendiendo por "estudio integral" el esfuerzo por descubrir tanto las interrelaciones funcionales existentes entre las

\footnotetext{
* Ponencia presentada al Seminario de Administración Educativa, realizado en Bogotá en abril de 1982.

Trabajadora Social, Universidad Externado de Colombia. Estudios de Postgrado en Investigación Socioeducativa Universidad Pedagógica Nacional. Investigadora del Centro de Investigaciones de la Universidad Pedagógica (CIUP)

${ }^{2}$ Marcel Mausa, Introducción a la Etnografía, Madrid, Ediciones Istmo, 1974.
} 
instituciones sociales encontradas en cada grupo humano específico, como las relaciones de aquellas con las necesidades básicas universales del hombre ${ }^{3}$.

La investigación antropológica, no ya de los pueblos "primitivos" y en trance de extinción, sino de sociedades "complejas" con presente y futuro en la historia, representó también un momento definitivo en el desarrollo de los trabajos etnográficos.

No solamente por los problemas metodológicos que planteaba el hecho de convertir lo cotidiano y familiar en el núcleo de la actividad investigativa, sino por la necesidad de compartir el objeto de estudio con otras tradiciones metodológicas (experimentales, estadísticas, etc.), la Etnografía debió perfeccionar sus técnicas de trabajo de campo, en particular la conocida como observación participante; adecuar las provenientes de otros métodos a sus propios intereses de investigación (entrevista, encuesta, etc.) enfatizar la naturaleza cualitativa y de estudio en profundidad como lo específico de su perspectiva frente a la de las otras modalidades metodológicas y reafirmar como propios y adecuados, aún en las nuevas circunstancias, los siguientes preceptos antropológicos fundamentales:

1)Abandonar las preconcepciones o estereotipos propios frente a los fenómenos sociales observados y explorar la manera como aquellos son vistos y construidos por sus participantes.

2) Convertir lo conocido en extraño, lo común en extraordinario, registrar lo que se da por hecho e indagar sobre las razones del por qué existe como es y no de otra manera.

3)Asumir que para comprender lo particular se necesita relacionarlo con su medio, con su contexto.

4) Utilizar la teoría social existente sobre el problema o el fenómeno estudiado para guiar la propia investigación ${ }^{4}$.

Este último precepto no invalida lo que, sin embargo, constituye también otro de los principios metodológicos de la Etnografía actual: la adopción de una actitud abierta y flexible de parte del investigador, que le permita concebir el trabajo investigativo como un proceso permanente de descubrimiento y comprensión de la realidad que estudia, de reflexión teórica sobre la misma, de construcción del objeto de investigación ${ }^{5}$ y de hallazgos inesperados que, así se traduzcan solamente en problemas nuevos para ser abocados en futuras investigaciones, enriquecen considerablemente el bagaje de conocimiento existente sobre el tema de estudio.

Con esta tradición y estos principios, la Etnografía asumió la tarea de tratar de comprender los fenómenos educativos en las sociedades contemporáneas.

La idea surgió en los Estados Unidos más o menos hacia los años 60 y en respuesta a la situación creada por el hecho de que, no obstante las cuantiosas sumas invertidas en investigación educativa orientada por los procedimientos corrientes de experimentación,

\footnotetext{
${ }^{3}$ Elsie Rockwell, La Relación entre Etnografía y Teoría en la Investigación Educativa, México, D.I.E. —IPN, 1980 (mimeo).

4 Kathleen Wilcox, The Ethnography of Schooling: Implications for Educational Policy-Making, University/Hayward, 1980 (mimeo).

${ }^{5}$ Elsie Rockwell, op. cit. 
encuesta y constitución de modelos matemáticos, los problemas identificados como prioritarios en el sistema educativo norteamericano no habían podido solucionarse aun.

Desde entonces la Etnografía educativa ha experimentado un desarrollo con múltiples y a veces contradictorios enfoques teóricos y metodológicos. Sin embargo, es posible precisar dos grandes líneas de investigación que, aunque no siempre compatibles, pueden interpretarse como momentos necesarios en el proceso de conformación y de consolidación de la propuesta etnográfica para la investigación educativa.

La Micro-etnografía que tiene ya una amplia trayectoria (más o menos 20 años) de contribución al conocimiento de los problemas educativos y la Macro-etnografía que data de una época más reciente y con muchos problemas para resolver aún.

\section{a) Micro-etnografía}

Los estudios micro-etnográficos centran su foco de atención en la institución educativa, y más concretamente en la situación de interacción maestro-alumno al interior del aula de clase.

Enmarcados en las formulaciones teóricas de la sociolingüística, interpretan el proceso educativo como un proceso de comunicación en el que la mayor o menor bondad de sus resultados puede ser explicada por la tesis del "conflicto cultural".

En efecto, la micro-etnografía ha tratado de demostrar que la interacción verbal y no verbal que se genera entre los participantes de una experiencia educativa en el salón de clase, no sólo es determinante en el éxito o el fracaso académico de los estudiantes, sino que a su vez depende, en su naturaleza y contenido, de la "competencia comunicativa" existente entre los actores.

En este sentido, el fracaso escolar no es más que el resultado previsible de una determinada forma de interacción en la que los dos tipos de actores (maestro y alumnos) provienen de tradiciones y contextos culturales diferentes.

El principal aporte de este tipo de estudios ha sido, no obstante, el de enriquecer él análisis de los hechos educativos con la contrastación entre lo normativo y deseable y lo que en efecto sucede al interior del aula de clase.

La micro-etnografía, por otra parte, ha tenido y tiene muy buena acogida entre las distintas instancias del sistema educativo en los países en los que se la ha adoptado como instrumento de evaluación. Las razones que explican este hecho son varias: a nivel de los sectores políticos y administrativos, los informes micro-etnográficos son de gran utilidad para implementar reformas que en ningún caso comprometen ni la totalidad ni una parte significativa de la estructura social, pero que sí han representado mayor eficacia en el logro de los objetivos previamente definidos para la institución educativa; a nivel de los establecimientos educativos y de los docentes, los resultados de los estudios microetnográficos constituyen una orientación valiosa cuando existe el interés de aprovecharlos para hacer más eficiente su labor; a nivel de las personas y entidades que investigan en educación porque, dadas sus posibilidades pragmáticas y los relativamente limitados recursos que demanda, la investigación microetnográfica resulta fácilmente financiable ${ }^{6}$.

\footnotetext{
${ }^{6}$ Ogbu, John U., School Ethnograph : A Multi-level Approach, University of California, D. of Anthropology, 1980 (mimeo).
} 


\section{b) Macro-etnografía}

La macro-etnografía aparece en cierta medida como reacción ante el reduccionismo característico de la micro-etnografía.

Aquella propone, un poco en el sentido de regresar a la concepción holística de la antropología clásica, enfocar el estudio de la Institución educativa en sus interrelaciones con las demás instituciones sociales, económicas, culturales y políticas presentes en una comunidad. Se trata de ver entonces de qué manera inciden una serie de factores históricos, socioeconómicos, ecológicos, etc., en el proceso educativo vivido en la escuela. Tal es el caso, por ejemplo, de los estudios realizados por Richard Warren en un pueblo alemán (1967) y por John Singleton en uno del Japón.

Ogbu propone ${ }^{7}$, como alternativa que permitiría superar las limitaciones tanto de la micro como de la macro-etnografía y a la vez aprovechar lo positivo de las dos, lo que ha denominado el modelo de múltiples niveles o método ecológico-cultural, cuyos supuestos básicos son:

1)La educación formal está relacionada de tal manera con las otras instituciones de la sociedad que la escuela puede afectar el comportamiento de la gente, en especial en las relaciones económicas.

2)El carácter de tal interacción tiene una historia que en cierto modo influye en los procesos educativos que existen actualmente.

3)El comportamiento de los participantes corresponde en alguna medida a su manera de ver la realidad social.

4) Para que una etnografía educativa sea adecuada, el estudio etnográfico no se puede basar exclusivamente en el estudio de los acontecimientos en la escuela o en el salón de clase. Debe incluir el estudio de las fuerzas comunitarias e históricas apropiadas.

Aunque la macro-etnografía y los diversos enfoques que parten de una consideración global o "integral" del proceso educativo, significan un progreso en el sentido de conocer y entender mejor la relación Educación y Sociedad, sus informes no siempre han sido bien recibidos en las esferas decisorias del sector educativo. Es evidente que cuando la investigación traspone los límites de la escuela, así no haya ido mucho más allá de los propios de una comunidad específica, como es el caso de los trabajos realizados bajo estas perspectivas, las posibilidades de intervenir a partir de sus recomendaciones disminuyen o se hacen mucho más complejas para los responsables de la formulación de política educativa. Por esta razón, las críticas más recurrentes dirigidas a las demandas o conclusiones provenientes de la investigación macro-etnográfica -y que comparte con las de toda investigación global del problema educativo-, pueden sintetizarse así: no tienen una utilidad práctica inmediata, en la medida en que señalan la necesidad de cambios en instituciones o sectores político-administrativos sobre los que los planificadores educativos no tienen ninguna injerencia, y cuya implementación implicaría una reforma más o menos radical de la estructura social.

La naturaleza de este cuestionamiento y el hecho de que, no obstante el mismo, se reconoce la contribución que pueden hacer los enfoques etnográficos holísticos a la

\footnotetext{
${ }^{7}$ Ibidem. 
formulación de políticas, pero en el largo plazo $^{8}$, hace pensar que la crítica debería dirigirse al fraccionamiento de los organismos y procesos de planificación, antes que a la factibilidad de las demandas de cambio de la etnografía.

Desde otro punto de vista ${ }^{9}$, en cambio, la macro-etnografía se reconoce como un avance en la comprensión del fenómeno educativo, aunque no sea todavía una solución adecuada para el problema central de la etnografía educativa actual: ¿cómo lograr una "descripción de la institución educativa, como instancia articulada orgánicamente a la estructura de la sociedad global?

En esta dirección se adelanta actualmente la reflexión teórica y metodológica sobre la Etnografía.

\section{Etnografía, Investigación y Planeación Educativa en Colombia}

La investigación educativa en Colombia, tanto básica como aplicada, es un campo relativamente reciente que, sin embargo, ha abarcado aspectos muy diversos del fenómeno educativo.

Hacer una enumeración exhaustiva de los mismos no es el objetivo de esta exposición. Se trata tan sólo de elaborar una clasificación general de los temas y problemas abordados, así como de las metodologías corrientemente empleadas para tal fin, con el propósito de contrastarlas con las posibilidades que en el mismo campo tendría la Etnografía. Las relaciones Economía y Educación, Educación y Sociedad y Educación y Cultura condensan en grandes áreas la temática de la investigación educativa del país, y los marcos teóricos de referencia obligatoria para el planeamiento educativo.

a)Las relaciones entre Economía y Educación constituyen el objeto específico de estudio de la Economía de la Educacion.

Entre los distintos problemas que esta disciplina ha contemplado, algunos exigen, y aportan al planeamiento educativo, conceptualizaciones y metodologías propias. Tal es el caso de los análisis sobre la rentabilidad individual y social de la educación, las demandas educativas del aparato productivo (estudios de recursos humanos), la interacción entre educación y empleo, educación y distribución del ingreso y educación y crecimiento económico.

Existe otro conjunto de temas que, por el contrario, requerirían del aporte cualitativo de los estudios de caso, ya como complemento, ya como sustitutos de las metodologías empleadas hasta hoy: los aspectos económicos del sistema educativo.

Los estudios sobre administración y financiación presupuestal, por ejemplo, se enriquecerían con el conocimiento de las características que la misma adquiere a nivel de distintas instituciones educativas. Los de costos con la consideración de la relación entre el costo de la unidad escolar y el contexto en el que ésta se inscribe. Una primera experiencia en este sentido la constituye el trabajo titulado "Costo Escolar y

\footnotetext{
${ }^{8}$ Wilcox, op. cit.

${ }^{9}$ Rockwell, op. cit. 
Mejoramiento Cualitativo de la Educación" ${ }^{10}$, conjunto de estudios de caso de seis escuelas rurales que llama la atención sobre la posibilidad y la necesidad de redefinir el concepto de costo escolar, y de elaborar una categoría que involucre todas las condiciones que determinan la operación y eficiencia de la escuela en distintos contextos socioeconómicos.

La Etnografía permite plantear, además, en esta como en las otras dimensiones del fenómeno eduix ${ }_{1}$ tivo, preguntas que no tienen cabida o respuesta apropiada en otro tipo de enfoque metodológico: ¿Qué valor social y económico tiene la educación para los distintos sectores sociales que acceden a ella? ¿Qué relación existe entre la naturaleza del trabajo que se realiza en la escuela y las distintas formas de producción económica propias de los contextos en los que aquella se ubica? ¿Qué valores y actitudes genera la escuela hacia el trabajo y los distintos tipos de trabajo en sus alumnos?

b)La Sociología de la Educación se ha preocupado a su vez por el análisis de la relación entre Educación y Sociedad.

El haber privilegiado hasta ahora los niveles macro del programa con el empleo de técnicas como la investigación por encuesta y el análisis estadístico de la información, le ha permitido centrarse en el examen de las vinculaciones generales entre educación y clase social, educación y movilidad social y educación y participación política.

El camino etnográfico sobre la posibilidad de entender aquella relación original en otra perspectiva: el cuestionamiento sobre las formas y modelos de participación de los individuos en la sociedad se plantea ahora desde la reflexión sobre el cómo vive la sociedad en el individuo, y a qué tipo de conductas y de interacción da lugar esa estructura de significados.

De allí que, en relación con la problemática específicamente educativa, la sociología se pregunte sobre el sentido que realmente tiene la escuela en la sociedad, y el significado contextual de categorías y conceptos fundamentales de la disciplina como proceso de socialización, función social de la escuela, condicionamientos socioeconómicos de la educación, imagen del maestro, características del rol docente, status de la profesión y las relaciones sociales al interior del proceso educativo, entre otros ${ }^{11}$.

c) En la comprensión de la relación existente entre Educación y Cultura es quizá donde tiene la Etnografía y en general la investigación educativa su más amplio campo de acción.

La carencia en algunos casos, y la insuficiencia de investigaciones en otros, referidas a dimensiones educativas esenciales para el conocimiento y la interpretación de la génesis y de los procesos constitutivos de la cultura nacional tales como el discurso y la

\footnotetext{
${ }^{10}$ Alba Paulsen de Cárdenas, Costo Escolar y Mejoramiento Cualitativo de la Educación, presentado al Seminario sobre Educación Rural organizado por el CIID en Bogotá, 1981. Revista Colombiana de Educación No. 8.

${ }^{11}$ Ver al respecto: Myriam Stella Ferro, Mi vida: Historia de la vida de una maestra rural colornbiana, Bogotá, América Latina, 1978; Juan Carlos Tedesco y Rodrigo Parra, Marginalidad

Urbano y Educación Formal, Buenos Aires, Proyecto Desarrollo y Educación en América Latina y el Caribe, UNESCO-CEPAL-PNUD, 1981; Rodrigo Parra y Leonor Zubieta, La Imagen del Maestro en la Escuela campesina, Bogotá, Centro de Investigaciones de la Universidad Pedagógica, 1981.
} 
práctica pedagógica o los contextos filosóficos, antropológicos e históricos de la realidad educativa en distintas épocas así lo hacen suponer ${ }^{12}$.

Naturalmente que la investigación histórica o la filosófica tienen sus propios procedimientos metodológicos, pero en la investigación de la actualidad pedagógica la Etnografía sí ocuparía un lugar privilegiado. De hecho ya se vienen adelantando algunos trabajos en esa perspectiva teórica y metodológica ${ }^{13}$.

\section{Etnografía y política educativa}

La Etnografía puede contribuir realmente a muy distintos niveles del planeamiento y de la formulación de políticas educativas. Los trabajos etnográficos, sea su orientación pedagógica, sociológica o antropológica, pueden proveer a la sociedad en su conjunto de una fundamentación empírica y de desarrollos teóricos y conceptuales que contribuyan a la descripción e Interpretación de la cultura. En términos más pragmáticos y por lo tanto de más inmediata utilidad, los trabajos de caso, en profundidad y cualitativos pueden aportar información y análisis pertinentes y necesarios para el proceso de toma de decisiones. Esto es especialmente válido si persiste el interés por el mejoramiento cualitativo de la educación como política prioritaria del sector educativo, y la identidad entre calidad y eficiencia (mayor promoción, mayor retención, menor repitencia, menor deserción) que sustenta la documentación oficial.

A ese propósito contribuirían, y con los límites ya señalados para la macro y la microetnografía en general, las investigaciones etnográficas sobre la práctica pedagógica en el aula, la organización social de la escuela, la implementación institucional de programas y reformas, la administración escolar, relaciones escuela-comunidad, relaciones maestro alumno, el fracaso escolar, evaluación cualitativa de los factores "internos" y "externos" que inciden en los resultados del proceso educativo, etc.

El que la metodología etnográfica pueda ir más allá, si bien depende del desarrollo teórico de diversas disciplinas que la informan o del cómo se resuelva el problema de la interpretación —última etapa metodológica-_ que tiene en la actualidad, fundamentalmente depende de las condiciones de cambio que efectivamente existan en la sociedad.

\footnotetext{
${ }^{12}$ A esta conclusión se llegó en el Seminario sobre el Estado del Arte de la Investigación Educativa celebrado en Bogotá en abril de 1980 y coordinado por el Instituto SER de Investigación. Ver en especial el informe presentado por Alberto Alvarado Acevedo.

${ }^{13}$ Araceli de Tezanos con la colaboración de Guillen Muñoz y Emiliano Romero en el marco del proyecto "Escuela y Comunidad en América Latina", Convenio CIID - CIUP, para el caso colombiano 